\title{
Fluid Motion Induced by Gravitational Differentiation of Immiscible Two Phases: Basic Equations and Linear Analyses
}

\author{
Shigeki KoBAYASHI ${ }^{1}$, Yutaka ABE $^{2}$, and Yoshio FUKAO ${ }^{1}$ \\ ${ }^{1}$ Department of Earth and Planetary Sciences, Nagoya University, Chikusa, Nagoya 464-01, Japan \\ ${ }^{2}$ Department of Earth and Planetary Physics, University of Tokyo, Bunkyo, Tokyo 113, Japan
}

(Received January 4, 1993; Revised June 7, 1993; Accepted June 7, 1993)

\begin{abstract}
Fluid motion caused by flotation of fine immiscible particles is investigated. Fine immiscible particles can be formed by several mechanisms in the Earth's outer core. For example, light materials may exsolve from cooling iron melt. Light-material-rich blobs may be formed through a boundary layer instability, even if light materials are thermodynamically miscible with molten iron. Governing equations for two-phase flow composed of fine particles and ambient fluids are derived from the conservation laws of mass and momentum. The stationary state without macroscopic fluid motion is neutrally stable to an infinitesimal disturbance. Transient fluid motion is induced by a horizontal disturbance of particle concentration. The resultant fluid motion can be classified into three types. One is a circulative motion driven by the buoyancy of fine particles and the others are non-circulative flows caused by "apparent compressibility" of two-phase flow. If we took into account effects of nonlinear advection, the buoyancy-driven flow would be self-sustaining. The relationship between the flow types and values of nondimensional parameters are investigated. The parameter range where the buoyancy-driven motion is observed is approximately given by $G>3 Q^{2}$ and $G>30 Q$. $G$ and $Q$ are the nondimensional parameters defined as $G \equiv \rho \delta H^{3} g / \eta^{2}$ and $Q \equiv \Delta v_{z} \rho H / \eta$, where $\rho, \eta, \delta, H, \Delta v_{z}$ and $g$ are the density and viscosity of mixture, the difference of intrinsic density between particles and the ambient fluid, the depth of the fluid layer, the ascending velocity of particles relative to the motion of ambient fluid, and the gravitational acceleration, respectively. The lower the relative velocity of ascending particles is, the more effectively the circulative motion occurs. The values of physical parameters estimated for the outer core fall in the range in which a buoyancy-driven motion should be observed.
\end{abstract}

\section{Introduction}

Two sources of buoyancy have been proposed as the driving force of convective motions in the Earth's outer core. One is thermal buoyancy. The other is compositional buoyancy caused by light materials expelled from the growing iron-rich inner core (e.g., BRAGINSKY, 1963). As the cause of geodynamo, compositional buoyancy has been preferred to thermal on the grounds of thermodynamical consideration for the conversion efficiency of thermal to kinetic energy (e.g., Braginsky, 1963; METChNiK et al., 1974; HewitT et al., 1975; Gubbins, 1977; Loper, 1978; FEARN and LOPER, 1981; LOPER and ROBERTS, 1983).

In most of the previous studies of compositional convection in the core, light materials are assumed to be miscible with ambient molten iron (Fig. 1(a)). In such a case, the microscopic transfer of light materials is controlled by diffusion, and thus the microscopic flux of the buoyancy source is proportional to the concentration gradient of light materials. This situation is exactly the same as that of thermal convection, in which microscopic heat flux is proportional to temperature gradient. Hence, the governing equations of compositional convection of this type are the same as those of thermal convection. Thus, we can expect that the properties of compositional convection of this type are similar to those of thermal convection. 
(a)

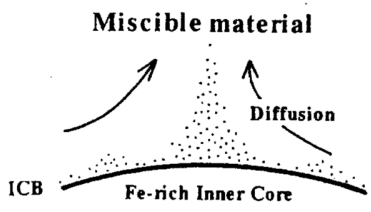

(c)

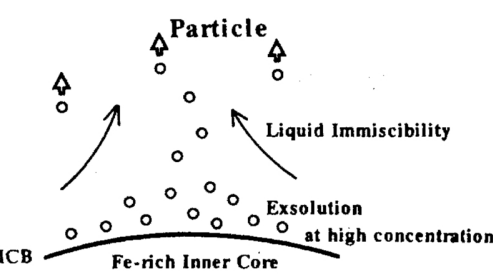

(b)

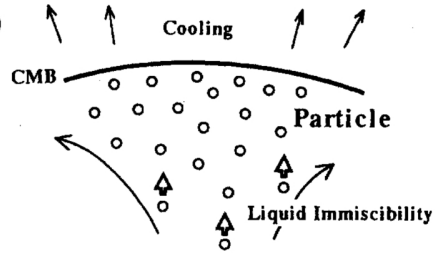

(d)

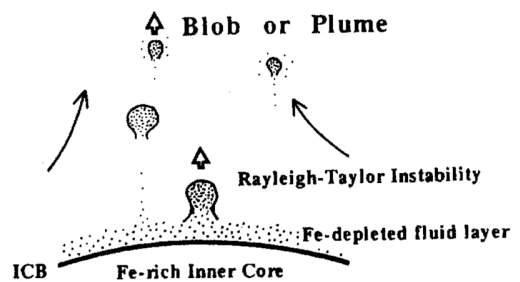

Fig. 1. Various possibilities on production of light elements in the outer core. (a) If light elements expelled from the growing iron-rich inner core are miscible with the ambient liquid iron, the convective motion induced by the buoyancy of light elements is essentially the same as that of thermal convection. (b) As the outer core cools, some immiscible light elements may exsolve from the molten iron alloy and float in the shape of fine "particles". (c) If light elements are expelled from the inner core at high concentration, they may not dissolve into iron alloy. In this case, many fine immiscible light element particles are formed and rise from the inner core boundary. (d) "Immiscible" light particles are also formed even if the light elements expelled from the inner core are miscible with the ambient molten iron in the thermodynamical sense. Owing to small molecular diffusivity, a buoyant iron-depleted fluid layer is formed at the bottom of the outer core. By a Rayleigh-Taylor instability, buoyant "blobs" or "plumes" are formed and rise in the outer core.

However, we can also imagine another type of compositionally driven convection, the flow induced by flotation of fine immiscible particles of light materials. Immiscible particles can be formed in the outer core in several ways. As the outer core cools, some light materials may exsolve from the molten iron alloy as immiscible liquid (Fig. 1(b)). Some researchers (e.g., VERHOOGEN, 1973; Ohtani and Ringwood, 1984; Urakawa et al., 1987; Kato and Ringwood, 1989) argued possible appearance of liquid immiscibility in the Fe-S, Fe-O or Fe-O-S system. High pressure experiments by ITO et al. (1992) suggest exsolution of silica-rich melt from the molten iron alloy during the cooling process of the outer core. Another possibility is related to growth of the inner core. If light materials are expelled from the growing inner core at too high a concentration, they may not dissolve into the molten iron alloy of the outer core. Thus, many fine immiscible particles of light materials are formed and rise from the inner core boundary to the core-mantle boundary (Fig. 1(c)). Effectively immiscible light particles can be formed even if they are thermodynamically miscible with the ambient molten iron. Owing to small molecular diffusivity, light materials expelled from the inner core tend to make a buoyant boundary layer at the bottom of the outer core, even if they are miscible with molten iron. Through an instability similar to the Rayleigh-Taylor instability, many "plumes" are formed (Fig. 1(d)) and, then, many "blobs" rise in the outer core (e.g., MofFATt, 1989). A similar situation is also suggested for a thermal convection with very high Rayleigh number, in which the molecular diffusion is essentially negligible (KIMURA, 1988).

If the fluid motion in the outer core is driven by flotation of light particles, it might be different from that of "ordinary" compositional convection induced by miscible light materials. For example, laboratory experiments revealed an interesting feature of the flow induced by flotation of bubbles. KIMURA (1988) observed flotation of the fine bubbles uniformly produced by electrolysis at the bottom of a fluid layer. He showed that circulative motion, a "bubble convection", is 
induced by flotation of fine bubbles. Moreover, he found that the horizontal scale of the bubble convection increases with increasing flux of bubbles.

There are many applications of "bubble convection", or the particle-fluid interaction problem, in Earth and planetary physics apart from convection of the outer core. The differentiation of protomantle, which is responsible for the layered structure of the terrestrial planets, is a result of sedimentation (or flotation) of crystals in a convecting magma ocean. Convection in magma chambers, which are directly connected to the problems of magma mixing and eruption, are modified by vesiculation or sedimentation of phenocrysts (e.g., KOYAGUCHI et al., 1990). The gravitational instability associated with the formation of planetesimals, the building blocks of planets, is also affected by interactions between sedimentation of dust particles and turbulent motion of nebular gas.

Bubble convection is also very interesting from a theoretical point of view. We introduce a concept of "transfer of buoyancy source" in fluid elements. The convective flow is driven by buoyancy force acting on fluid element. In the case of thermal convection, for example, the buoyancy force is caused by the temperature heterogeneity in the fluid. In this case, we consider temperature anomaly is the source of buoyancy. Heat conduction in the fluid changes distribution of buoyancy source through changing temperature distribution. Hence, we may concider that buoyancy source is transfered by heat conduction. Similarly, in the case of ordinaly compositional convection, buoyancy source is the anomaly in concentration of light components, and it is transfered by molecular diffusion in the fluid. In the case of bubble convection, the buoyancy source is again the anomaly of particle concentration in the fluid, but it is transfered by ascending motion of particles relative to the ambient fluid. We should note the difference in the mechanism of microscopic transfer of buoyancy source between the bubble convection and thermal or ordinary compositional convections. In the case of bubble convection, immiscible light particles are always rising relative to the ambient fluid irrespective of their concentration gradient. Hence, the microscopic flux of buoyancy sources in the bubble convection is not related to their concentration gradient, but it is proportional to their concentration (see also Section 3). On the other hand, in the case of thermal or ordinary compositional convection, flux of buoyancy source is controlled by diffusion and proportional to their concentration gradient. Thus, the mechanism of microscopic transfer of buoyancy source in bubble convection is completely different from the one in thermal convection or compositional convection driven by miscible materials. Such a difference could be very important because the microscopic transfer of buoyancy source is related to the stability of the fluid layer. For example, if there are no mechanisms of microscopic transfer of buoyancy sources, the fluid layer is always unstable to the injection of lighter materials in the lower part of the layer, in a sense of the Rayleigh-Taylor instability. If, on the other hand, transfer of buoyancy sources is proportional to their concentration gradient, the fluid layer is lead to the Rayleigh-Bénard instability. Our question is addressed to what type of instability appears if the microscopic transfer of buoyancy source is controlled by its concentration (bubble convection).

The purpose of this study is to obtain a better understanding of the characteristics of the fluid motion caused by flotation and/or sedimentation of fine particles, or bubble convection, and such a fluid motion. First, we derive the governing equations for two-phase flow composed of fine particles and ambient fluid. Then, we analyze the linear stability of the fluid layer and the flow pattern caused by flotation of particles by using a linearized version of the governing equations. Finally, an application to the core is discussed.

\section{Mathematical Formulation of the Problem}

\subsection{Basic concepts and assumptions}

We derive governing equations for two-phase flow composed of fine immiscible particles (henceforth, particles) and ambient fluid. We consider a case when particles are not so small 
as to be suspended in ambient fluid. Thus, buoyant particles are always rising relative to the ambient fluid. Relative motion between two phases plays an important role in this system. For simplicity, let us start with the following assumptions:

1) Both particles and the ambient fluid are incompressible.

2) The concentration of particles is small, so that the interactions between particles can be ignored.

3) The particles are spheres with a uniform size.

4) The relative velocity of each particle and the ambient fluid is slow enough so that the Stokes approximation can be applied to the flow around each particle.

We deal with the fluid motion in a much larger scale than the particle size, such that a fluid element involves many fine particles. Therefore, we formulate the change in the averaged quantity of particles involved in an element rather than describing the behavior of each particle.

\subsection{Derivation of the governing equations}

We denote the particles by phase $\alpha$ and the ambient fluid by phase $\beta$. The general forms of conservation of particles and the ambient fluid mass are written as follows (DE GROOT and MAZUR, 1962):

$$
\begin{gathered}
\dot{\rho}^{\alpha}+\nabla_{l}\left(\rho^{\alpha} v_{l}\right)+\nabla_{l} J_{l}=0, \\
\dot{\rho}^{\beta}+\nabla_{l}\left(\rho^{\beta} v_{l}\right)-\nabla_{l} J_{l}=0
\end{gathered}
$$

where $\rho^{\alpha}, \rho^{\beta}$ are, respectively, the masses of $\alpha$ and $\beta$ phases per unit volume of mixture and total density of mixture, $\rho=\rho^{\alpha}+\rho^{\beta}$. The barycentric velocity of mixture, $v_{i}$, is defined by

$$
\rho v_{i}=\rho^{\alpha} v_{i}^{\alpha}+\rho^{\beta} v_{i}^{\beta}
$$

where $v_{i}^{\alpha}$ and $v_{i}^{\beta}$ are their corresponding phase-averaged velocities and superscript $i$ means a component of $i$-th direction. We define

$$
\rho^{\alpha}=c \rho^{\alpha *}, \quad \rho^{\beta}=(1-c) \rho^{\beta *}
$$

so that $\rho^{\alpha *}, \rho^{\beta *}$ are the intrinsic densities of particles and fluid, respectively, and $c$ is the volume fraction of particles, or the concentration. The quantity $J_{i}$ is the mass flux of particles per unit area and time with respect to the barycentric motion, which is defined by

$$
J_{i} \equiv \rho^{\alpha}\left(v_{i}^{\alpha}-v_{i}\right) .
$$

By using relative velocity, $\Delta v_{i}\left(=v_{i}^{\alpha}-v_{i}^{\beta}\right)$, of particles with respect to the ambient fluid, $J_{i}$ is rewritten as follows

$$
J_{i}=\frac{\rho^{\alpha *} \rho^{\beta *}}{\rho} c(1-c) \Delta v_{i}
$$

The equation of motion for the mixture of particles and ambient fluid is derived from momentum balance.

$$
\rho\left(\dot{v}_{i}+v_{l} \nabla_{l} v_{i}\right)=-\nabla_{i} p+\nabla_{i} \sigma_{l i}^{\prime}+\rho g_{i}
$$

where $\sigma_{l i}^{\prime}$ and $p$ are the viscous stress tensor and the pressure of mixture, respectively, and $g_{i}(=(0,0,-g))$ is the gravitational acceleration.

The motion of the mixture is described by Eqs. (1), (2) and (7). Since we are interested in the motion of the ambient fluid rather than that of the mixture, we rewrite these equations by using fluid velocity, $v_{i}^{\beta}$.

$$
\begin{gathered}
\nabla_{l} v_{l}^{\beta}+\Delta v_{l} \nabla_{l} c=0 \\
\dot{c}+v_{l}^{\beta} \nabla_{l} c=-c \nabla_{l} v_{l}^{\beta}-\Delta v_{l} \nabla_{l} c
\end{gathered}
$$




$$
\rho\left(v_{i}^{\beta}+v_{l}^{\beta} \nabla_{l} v_{i}^{\beta}\right)+\rho^{\alpha *} c \Delta v_{l} \nabla_{l} v_{i}^{\beta}-\Delta v_{i} \nabla_{l} J_{l}=-\nabla_{i} p+\nabla_{i} \sigma_{i l}^{\prime}+\rho g_{i} .
$$

One should note that the flow field is compressible (i.e., $\nabla_{l} v_{l}^{\beta} \neq 0$ ), even if we assume that both phases are incompressible. This is an "apparent" compressibility caused by relative motion between two phases. This system is not compressible in a sense that the density dose not depend on pressure. Therefore, we do not have sound waves in this "apparent" compressible system. Regardless of their concentration gradient, buoyant particles are always rising relative to the ambient fluid microscopically. Hence, when the vertical distribution of particles is not uniform, the density of the mixture changes owing to the relative motion between the two phases.

\subsection{The stokes approximation}

Under the assumptions 2) and 4), we can apply the Stokes approximation to the (microscopic) flow field around each particle. In this approximation the relative velocity $\Delta v_{i}$ is the terminal velocity given by the following formula (BATCHELOR, 1967):

$$
\Delta v_{i}=\frac{2 a^{2}\left(\eta^{\alpha}+\eta^{\beta}\right)}{3 \eta^{\beta}\left(3 \eta^{\alpha}+2 \eta^{\beta}\right)}\left(\rho^{\alpha *}-\rho^{\beta *}\right) g_{i}
$$

where $\eta^{\alpha}$ and $\eta^{\beta}$ are the viscosities of particles and the ambient fluid, respectively; and $a$ is the radius of particles. Note that we consider spherical particles (assumption 3). For simplicity, we ignore the rotation of particles. In this case, the stress tensor is symmetric. In addition, the relative motion has no effect on a viscous stress tensor, under the Stokes approximation. Then, we can adopt the viscosity of suspensions (EINSTEIN, 1906; BATCHELOR, 1967) as an effective viscosity of the mixture, which is given by

$$
\eta=\left[1+\frac{5 \eta^{\alpha}+2 \eta^{\beta}}{2\left(\eta^{\alpha}+\eta^{\beta}\right)} c\right] \eta^{\beta} .
$$

Equations (11) and (12) are appropriate so long as the concentration of particles is sufficiently small.

\section{Linear Analysis}

\subsection{Perturbation equations}

Consider a quiescent layer of fluid with infinite horizontal extent and buoyant particles of uniform size generated homogeneously at the bottom. We take the $z$-axis in the vertical upward direction and the $x$ and $y$ axes in the horizontal directions. Since we assume a uniform particle size and terminal velocity, the particles rise at constant velocity $\Delta v_{z}$. It is clear from Eqs. (8)-(10) that there is a stationary state such that

$$
c=c_{0}=\text { constant }, \quad v_{i}^{\beta}=0 .
$$

In this state no (macroscopic) motion of the ambient fluid occurs, and the upward mass flux of particles is constant throughout the layer. This state corresponds to the stationary conductive state of thermal convection.

We analyze the stability of this stationary state against infinitesimal perturbations in particle concentration, $c^{\prime}$. We obtain a set of linearized equations from Eqs. (8)-(10) by ignoring terms with the second or higher order perturbations.

$$
\begin{gathered}
\nabla_{l} v_{l}^{\beta \prime}+\Delta v_{l} \nabla_{l} c^{\prime}=0, \\
\dot{c}^{\prime}=-\left(1-c_{0}\right) \Delta v_{z} \nabla_{z} c^{\prime},
\end{gathered}
$$




$$
\rho_{0} \dot{v}_{i}^{\beta \prime}+\rho^{\alpha *} c_{0} \Delta v_{z} \nabla_{z} v_{i}^{\beta \prime}=-\nabla_{i} p^{\prime}+\eta_{0} \nabla_{l} \nabla_{l} v_{i}^{\beta \prime}+\frac{1}{3} \eta_{0} \nabla_{i} \nabla_{l} v_{l}^{\beta \prime}+\left(\rho^{\alpha *}-\rho^{\beta *}\right) c^{\prime} g_{i}
$$

where the subscript 0 indicates stationary state quantities and 'indicates perturbation quantities.

Length and time are normalized by the depth of the fluid layer, $H$, and the "viscous diffusion time", $H^{2} \rho_{0} / \eta_{0}$. We obtain a set of nondimensional equations:

$$
\begin{gathered}
\nabla_{l} v_{l}^{\beta \prime}+Q \nabla_{z} c^{\prime}=0, \\
\dot{c}^{\prime}=-\left(1-c_{0}\right) Q \nabla_{z} c^{\prime}, \\
\dot{v}_{i}^{\beta \prime}+\left[1+D\left(1-c_{0}\right)\right] c_{0} Q \nabla_{z} v_{i}^{\beta \prime}=-\nabla_{i} p^{\prime}+\nabla_{l} \nabla_{l} v_{i}^{\beta \prime}-\frac{1}{3} Q \nabla_{i} \nabla_{z} c^{\prime}-G c^{\prime} \delta_{i z} .
\end{gathered}
$$

These equations contain three nondimensional parameters besides $c_{0}$ :

$$
G \equiv \frac{\rho_{0}\left(\rho^{\alpha *}-\rho^{\beta *}\right) H^{3} g}{\eta_{0}^{2}}, \quad Q \equiv \frac{\Delta v_{z} \rho_{0} H}{\eta_{0}}, \quad D \equiv \frac{\rho^{\alpha *}-\rho^{\beta *}}{\rho_{0}}
$$

where $G$ is a measure of buoyancy which corresponds to the Grashof number in thermal convection. $Q$ is the nondimensional relative velocity which corresponds to the inverse of Prandtl number in thermal convection. It also corresponds to the Reynolds number defined by the velocity of ascending particles and the depth of the layer. $D$ is the nondimensional density difference. The Rayleigh number in thermal convection is given by the product of the Grashof number and the Prandtl number. Therefore, we define a "pseudo-Rayleigh number" by $R b \equiv G / Q$.

\subsection{Linear stability}

Equation (15) has a general solution of the following form:

$$
c^{\prime}=f[x, y] h\left[\left(1-c_{0}\right) \Delta v_{z} t-z\right] .
$$

This means that the disturbance of particle concentration migrates upward with a constant velocity $\left(1-c_{0}\right) \Delta v_{z}$ without changing its shape. In other words, the disturbance shows neither growing nor decaying. The stationary state is, therefore, neutrally stable with respect to infinitesimal disturbances.

This neutral stability does not imply that no fluid motion is induced by the disturbance in particle concentration. Flow is induced. However, the induced flow does not modify the particle distribution because the effect of particle advection appears only as a nonlinear effect in this system.

It would be intriguing to compare this situation to thermal convection, in which the stationary state without fluid motion (i.e., conductive solution) has a vertical density gradient caused by a vertical temperature gradient. This vertical density gradient is an inevitable consequence of non-zero conductive heat flux. A small vertical displacement in such a stratified layer results in a horizontal density variation and, hence, buoyancy, which causes instability in the thermal convection. In bubble convection, on the contrary, the vertical mass flux of particles is proportional to the particle concentration (see Eq. (6); $J_{i} \propto c$ if $c \ll 1$ ). Hence, in a stationary state with a constant particle flux there is no vertical gradient in particle concentration, and thus, in density, where a vertical displacement of the fluid mixture does not create buoyancy. This is the physical reason why the stationary state with a constant mass flux of particles is neutrally stable.

Even in this case, however, finite-amplitude disturbances in particle concentration would induce non-zero fluid motion. If a sufficiently strong horizontal flow is induced, the flow would advect particles in horizontal direction, and a horizontal heterogeneity of particle concentration could be formed. This horizontal disturbance in particle concentration causes a horizontal density disturbance, which results in buoyancy torque in the fluid. Hence, if a sufficiently large horizontal flow is induced by a finite-amplitude disturbance in particle concentration, the flow could be selfsustaining. 


\section{Time-Dependent Numerical Solutions of the Linear Equations}

\subsection{Flow patterns}

We investigate fluid motion caused by a horizontally periodic disturbance of particle concentration by using linearized equations (17)-(19). We consider a two-dimensional fluid layer of depth $H$ with horizontally periodic boundary conditions:

$$
\begin{gathered}
v_{z}^{\beta \prime}=0 \quad \text { and } \quad \partial v_{x}^{\beta \prime} / \partial z=0 \quad \text { on } z=0, H, \\
c^{\prime}=0 \quad \text { on } z=0 .
\end{gathered}
$$

The normal velocity of the fluid and the shear stress vanish at both the top and bottom boundaries of the layer. The particle concentration is kept constant at the bottom. Since particle motion is not modified by the fluid flow in linearized system (see Eq. (17)), particles ascend at a constant velocity and finally escape from the top of the layer. Hence the constant particle concentration is equivalent to the constant mass flux of particles at both the top and bottom boundaries.

Figure 2 shows the initial distribution of particles: a horizontally periodic disturbance in particle concentration with wavelength $2 \lambda$ is given in the lower part of the layer. The initial velocity of the fluid is zero (i.e., no motion).

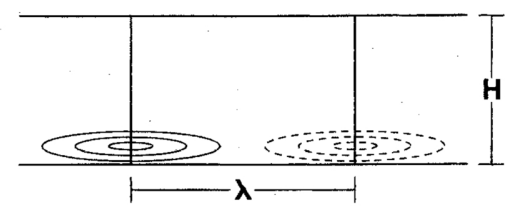

Fig. 2. Initial distribution of particles in the fluid layer. We consider horizontally periodic disturbances of particle concentration with wavelength $2 \lambda$ in the lower part of the fluid layer of depth $H$. Solid and broken curves are the contours of the positive and negative disturbances of particle concentration, respectively.

Development of the fluid motion is investigated numerically. A finite difference method is used in the vertical direction and a spectral method is used in the horizontal direction. We use the backward Euler scheme for time marching. As we shall see later in the next section, the regime of flow patterns is specified by the nondimensional parameters. Fluid motions induced by flotation of particles are classified into three types based on their flow patterns as summarized in the following.

(a) Type I flow: Figure 3 shows the development of the type I flow. This flow is marked by a cellular circulation or a convective motion which extends through the whole layer. Upward flow is induced at the region of high particle concentration. This indicates that the flow is caused by buoyancy of particles.

(b) Type II flow: Figure 4 shows the development of the type II flow. This flow is characterized by a localized vertical flow. Note that the flow direction is opposite to that of the type I flow; downward flow occurs at high particle concentration. Furthermore, this flow does not extend through the whole layer but confines itself in the region of the concentration disturbance. All these features indicate that the flow is not induced by buoyancy of particles but occurs to compensate the density change caused by migration of particles relative to the ambient fluid. In other words, this flow is a result of the apparent compressibility of the fluid mixture discussed in the previous section.

(c) Type III flow: Figure 5 shows the development of the type III flow. This is a horizontal one-way flow that develops at the moment when the concentration disturbance escapes from the 


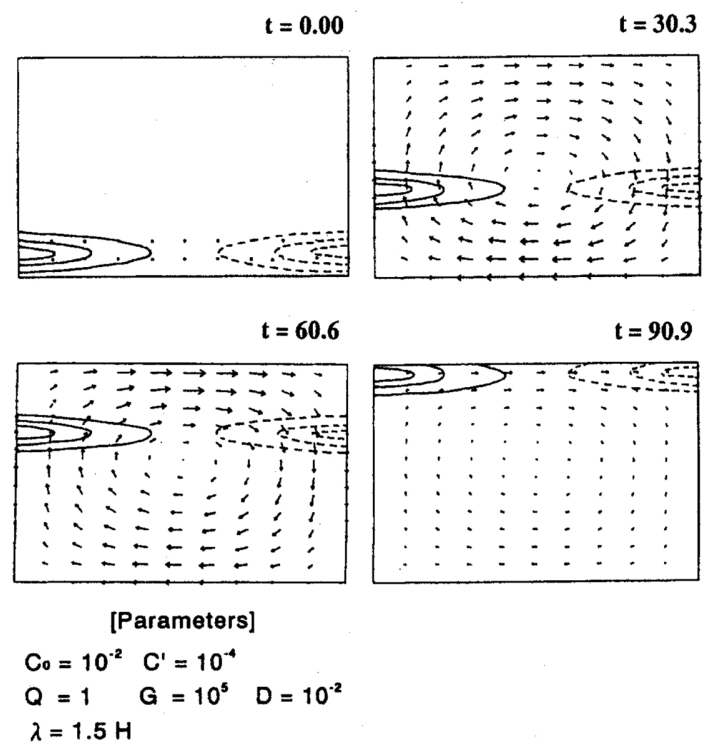

Fig. 3. Development of the type I flow. Small arrows indicate the velocity vector of the induced flow in the ambient fluid. Thin solid and broken curves are the contours of the positive and negative disturbances of particle concentration, respectively. As the disturbances migrate upward, cellular circulation is induced. Note that the upward flow occurs for a positive disturbance of particle concentration.

top of the fluid layer. This horizontal flow occurs to compensate the density change caused by loss of particles at the top of layer. Thus, the type III flow is also a flow caused by the apparent compressibility. It should be noted that its occurence is due to the boundary condition at the top of the layer; if light particles are not lost at the top of the layer, the flow of this type does not occur.

\subsection{Flow type selection}

We investigated the relationship between the flow types and the values of non-dimensional parameters-buoyancy $G$, relative velocity $Q$, density difference $D$, initial concentration of particles, $c_{0}$, and the horizontal half-wavelength of disturbance, $\lambda / H$. As is clear from Eq. (19), the parameter $D$ has little effect on the flow patterns if $D \ll 1$. Similarly, $c_{0}$ value does not change the flow patterns significantly as long as $c_{0} \ll 1$. Thus we shall focus our attention on the dependence of the flow patterns on the values of $G, Q$ and $\lambda / H$.

Figure 6 shows a "phase diagram" of flow types. This diagram shows the effect of a horizontal half-wavelength of disturbance, $\lambda$, and the non-dimensional relative velocity, $Q$, on the selection of flow type. In this diagram other non-dimensional parameters are fixed $\left(G=10^{5}, D=10^{-2}\right.$, and $c_{0}=10^{-2}$ ). Circles, triangles and squares in the diagram indicate conditions in which type I, II and III flows are observed, respectively. Thick lines are the inferred "phase boundary" of the flow types. At a small relative velocity, $Q$, the type I (convective) flow appears. On the other hand, at a large relative velocity either the type II or III flow appears. The type II (localized vertical) flow appears when the wavelength of disturbance is short $(\lambda<H)$. When the wavelength of the disturbance is long $(\lambda>H)$, the type III (horizontal one-way) flow appears.

Only the type I flow is driven by buoyancy. Hence, this diagram indicates that the buoyancy of particles can drive the convection of a whole layer (type I flow) only when the relative velocities of the particles are small. When the relative velocity is higher than a certain critical value, $Q_{c}$, 

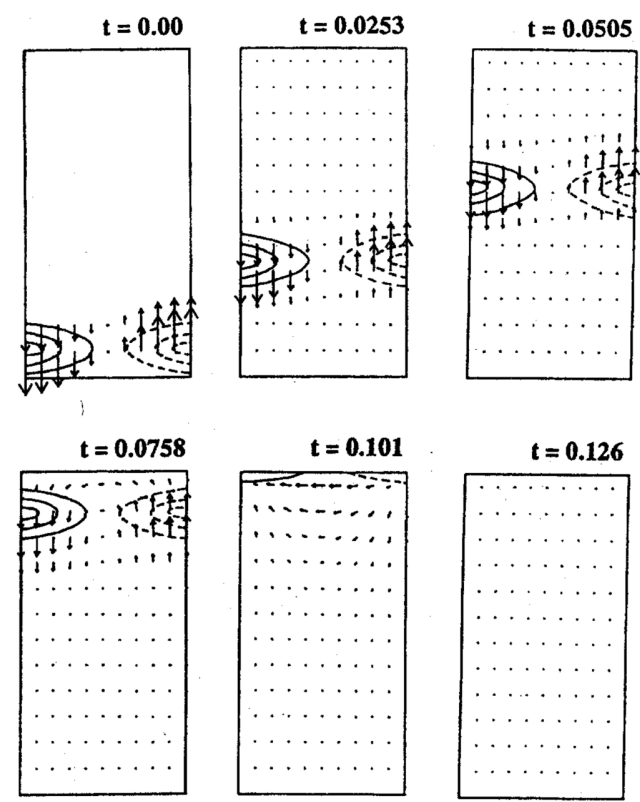

[Parameters]

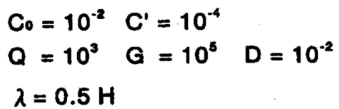

Fig. 4. Development of the type II flow. Symbols are the same as those of Fig. 3. Localized vertical flow is induced. The vertical flow is confined in the disturbed region of particle concentration. Note that the flow direction is opposite to that of the Type I flow; downward flow occurs at the positive disturbances of particle concentration.

(in this case $Q_{c}=10^{3}$ ), the buoyancy of the particles never drives convective motion.

This critical value, $Q_{c}$, is strongly dependent on $G$ value. Figure 7 shows the "phase diagram" of flow types plotted on a $G-Q$ plane. Here, the horizontal half-wavelength, $\lambda$, is fixed to $H$. The shaded region indicates the parameter range in which type I (convective) flow is observed. This figure shows that large buoyancy $(G)$ is required for inducing a buoyancy-driven motion when the relative velocity of ascending particles $(Q)$ is large. Alternatively, we may say that the critical $Q$ value for the buoyancy driven flow increases with increasing $G$ values. At large $G$ values $(G>300)$, the $Q_{c}$ value is approximately proportional to $\sqrt{G}\left(Q_{c} \approx \sqrt{G / 3}\right)$. At small $G$ values $(G<300)$, the $Q_{c}$ value is approximately proportional to $G\left(Q_{c} \approx G / 30\right)$. Furthermore, type I flows show essentially the same pattern if the $G / Q^{2}$ value is the same while $G>300$. Similarly, at small $G$ values $(G<300)$, type I flows show essentially the same pattern if the $G / Q$ value is the same. On the contrary; the type II and III flow patterns are determined only by $Q$ and independent of $G$. Independence of type II or III patterns to $G$ value is just anticipated from their non-buoyancy-driven nature.

These phenomena may be qualitatively understood by considering that a slower relative velocity gives a longer "residence time" of particles in a fluid element, and thus a stronger coupling of particles and ambient fluid. It may also be understood from the analogy to thermal convection. Suppose in a thermal convection system that the Grashof number, $G r$, is given. Since a larger Prandtl number, $P r$, results in a larger Rayleigh number, $R a(=G r P r)$, there is a critical Prandtl number for the onset of thermal convection. Thermal buoyancy does not drive convective motion, 


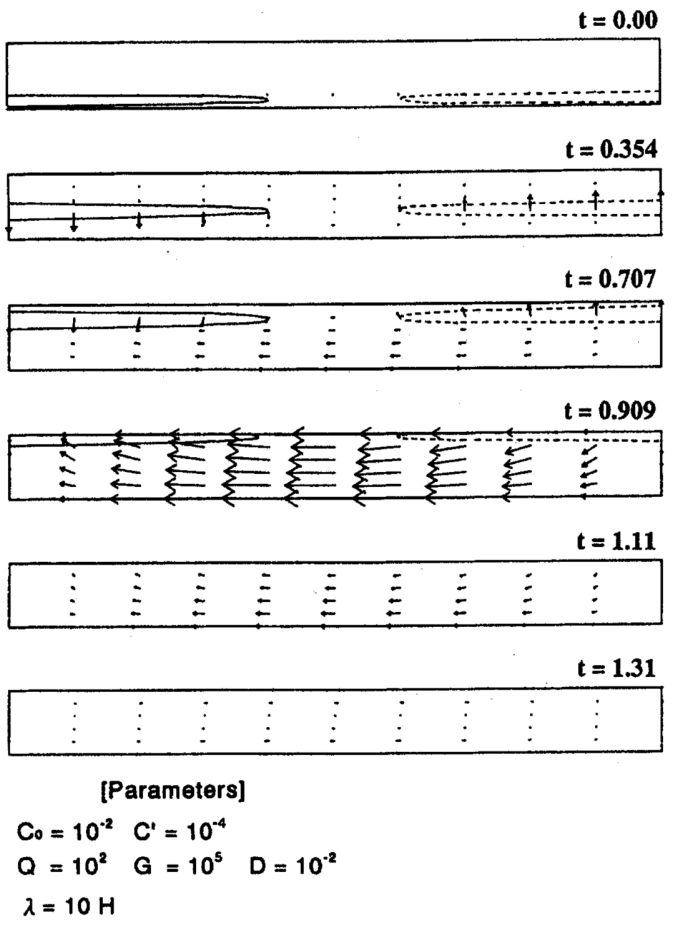

Fig. 5. Development of the type III flow. Symbols are the same as those of Fig. 3. Horizontal flow is induced when the particle concentration disturbance reaches the top of the fluid layer.

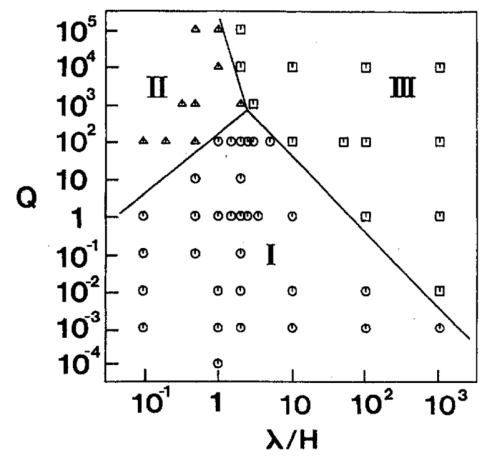

Fig. 6. The "phase diagram" of flow types. Vertical axis is the non-dimensional velocity of ascending particles. Horizontal axis is the horizontal wavelength of the disturbances normalized by the thickness of the fluid layer. Small circles, triangles and squares in the diagram indicate the parameter values at which the Type I, II and III flows are observed, respectively. 


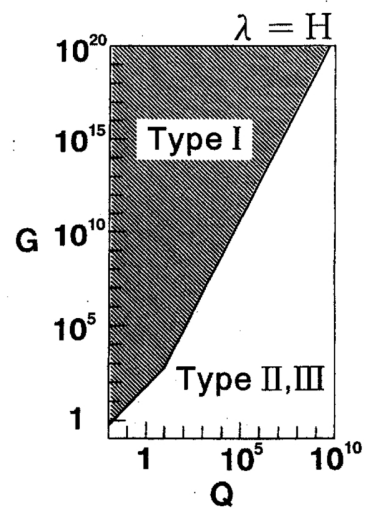

Fig. 7. The "phase diagram" of flow types plotted on $G-Q$ plane. Horizontal half-wavelength, $\lambda$, is fixed to $H$. Shaded region indicates the parameter range in which the Type I flow is observed. The shaded region is approximately given by $G>3 Q^{2}$ and $G>30 Q$.

if the Prandtl number is smaller than the critical value. In a bubble convection system, as noted before, $Q$ corresponds to the inverse of the Prandtl number, and the presence of a critical Prandtl number suggests the presence of a critical $Q$ value.

Such an analogy leads us to expect the existence of a critical "Rayleigh number" for our system. As noted before, $G$ and $Q$ correspond to the Grashof number and the inverse of the Prandtl number, respectively. Hence, we can define a "pseudo-Rayleigh number" by $R b \equiv G / Q$. Figure 8 shows the "phase diagram" of flow types plotted on a $R b-Q$ plane. Here, the horizontal half-wavelength, $\lambda$, is fixed to $H$. The shaded region indicates the parameter range in which Type I (convective) flow is observed. This figure suggests the existence of a critical $R b$ value for the buoyancy-driven Type I flow. The lower limit of the critical value, $R b_{c}$ is about 30 . The $R b_{c}$ value is approximately proportional to the $Q$ value $\left(R b_{c} \approx 3 Q\right)$ if $Q>10$. At present, we cannot derive the critical value and its dependence on $Q$ quantitatively based on the governing equations.

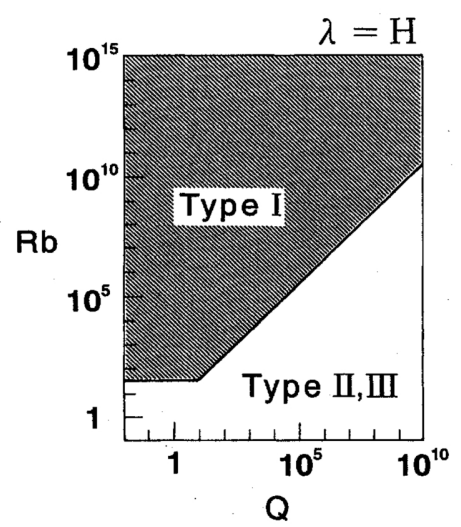

Fig. 8. The "phase diagram" of flow types plotted on $R b-Q$ plane. Horizontal half-wavelength, $\lambda$, is fixed to $H$. $R b$ is the pseudo-Rayleigh number defined by $R b=G / Q$. Shaded region indicates the parameter range in which the Type I flow is observed. The Type I flow appears only in the region $R b>30$, suggesting the existence of a critical $R b$ value for the Type I flow (critical value is about 30 ). 


\section{Concluding Remarks}

\subsection{Onset of convective instability}

This paper has been mainly concerned with derivation of the basic equations and linear analysis of the fluid motion induced by flotation of particles. The stationary state in this system is neutrally stable within the framework of the linear theory, because the fluid motion does not affect the distribution of particles in linearized dynamics. However, it does not imply that convective motion cannot be self-sustained by flotation of particles, because particle distribution is modified by flow through nonlinear advection. If the induced flow is strong enough to modify the particle distribution, a self-sustaining convective motion may take place. On the contrary, if the induced flow is too weak, the flow cannot be self-sustaining and must be transient. Therefore, induced flow strong enough to advect particles is a necessary (but not a sufficient) condition for self-sustaining convective motion. We can evaluate the efficiency of particle advection by comparing the horizontal velocity of induced flow and the ascending velocity of particles relative to the ambient fluid. If the former is larger than the latter, we can expect a large advection effect.

Figure 9 is a contour map of the ratio of the maximum horizontal velocity of the induced flow and the vertical velocity of ascending particles. The axes are the same as those of Fig. 6 and thick lines indicate the boundaries of the flow types. Numbers attached to the curves indicate the ratio of the induced flow velocity and the vertical velocity of ascending particles. The hatched region indicates the parameter range where the ratio is larger than unity. Particle distribution should be greatly modified by nonlinear advection in this parameter range where the linear analysis would lose its validity.

One should note, however, that horizontal velocity is proportional to the amplitude of the disturbed concentration of particles. On the contrary, the velocity of ascending particles is almost independent of the amplitude of disturbance. Hence, the ratio of flow velocity and particle velocity is proportional to the amplitude of the disturbance. In Fig. 9, the amplitude of the disturbance is assumed to be $10^{-4}$. The hatched region shrinks with decreasing amplitude of the disturbance.

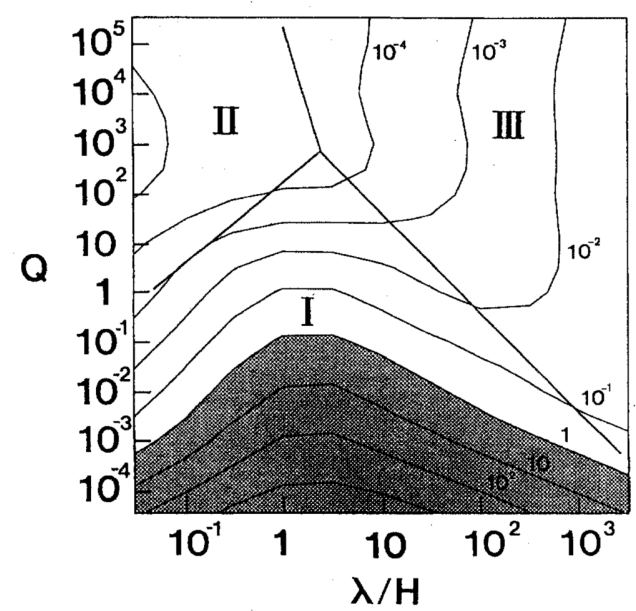

Fig. 9. Contour map of the horizontal velocity of the induced flow. The axes are the same as those of Fig. 6 . Thick lines indicate the boundary of the flow types. The horizontal velocity is normalized by the velocity of the upward migration of particles. Numbers attached to the curves indicate the ratio of the induced flow velocity and the velocity of ascending particles. Hatched region indicates the parameter range where the induced flow velocity is larger than the velocity of ascending particles. Significant horizontal advection of particles occurs in this region. 
At the limit of an infinitesimal disturbance the hatched region disappears. This is consistent with the result of the linear stability analysis which indicates neutral stability.

Note that the hatched region is confined in the range of the type I flows. Although the hatched region extends with increasing amplitude of the disturbance, it never extends to the region of the type II or type III flows, where the amplitude of the disturbance never exceeds unity. This indicates that only the type I flows, the buoyancy driven convective motion, can develop into a self-sustaining flow. The type II or III flows cannot be self-sustaining. The flow pattern will be significantly affected by the nonlinear advection in the hatched region. Hence, in such parameter ranges, we need a full nonlinear analysis to understand the fluid motion. Nonlinear behavior of the type I flow is under intensive investigation by the authors and the results will be reported elsewhere. The type II or III flows, which appear outside the hatched region, would not be so significantly affected by the nonlinear advection effect as the type I flow. Hence, these flows are well described by the present linear analysis.

\subsection{Implication for the core convection}

The present analysis is not directly applicable to the core convection. In the real core we must take into account the effects of thermal buoyancy, rotation and the magnetic field. Moreover, it is not clear whether or not the light materials behave as immiscible particles in the outer core. The most important issue at this moment is to discriminate the flow fields between the miscible and immiscible cases. For the full discrimination we have to await the results of nonlinear analysis; the particle-driven convection is essentially a nonlinear problem unlike the thermal convection case.

Nevertheless, it is interesting to see what kind of fluid motion could be induced in the outer core by flotation of immiscible light materials. Suppose that the viscosity of light materials is the same as that of the ambient core fluid, the density of the fluid is $10 \mathrm{~g} / \mathrm{cm}^{3}$, and the density difference between the molten iron alloy and the light materials is $0.1 \mathrm{~g} / \mathrm{cm}^{3}$. The depth of fluid layer, $H$, is put equal to the thickness of the outer core. Since the gravity is $5 \mathrm{~m} / \mathrm{s}^{2}$, $G=6 \times 10^{25} / \eta^{2}$ and $Q=2 \times 10^{12} a^{2} / \eta^{2}$ where $a(\mathrm{~m})$ is the radius of particles. The viscosity of the core, $\eta$ (Pas), is not known precisely and the estimated values largely scatter in a range between $10^{-4}$ and $10^{9}$ (Pas) (e.g., JACOBS, 1975; PoIRIER, 1988). In a whole range of the estimated viscosity, expected values of $G$ and $Q$ fall in the region of the type I flow, if $a<10^{-2}$ (m) (Fig. 7). FrancK (1982) estimated the possible size of immiscible particles based on an argument of surface tension and showed that the particles grow up to a radius of about $6 \times 10^{-5}$ $(\mathrm{m})$ and then begin to float. Thus, a convective motion is possibly sustained by the flotation of immiscible light materials in the outer core. In this case, the velocity of the flow depends on the particle size as well as the mass flux of light materials.

Thanks are due to Professor Ryuji Kimura (Ocean Research Institute) and an anonymous reviewer for helpful suggestions and comments on improving the manuscript.

The numerical calculations were made at the Nagoya University Computer Center. This research was partially supported by the Grant-in-Aid for Scientific Research (No. 03232103) from the Ministry of Education, Science and Culture of Japan.

\section{REFERENCES}

Batchelor, G. K., An Introduction to Fluid Dynamics, 615 pp., Cambridge Univ. Press, 1967.

Braginsky, S. J., Structure of the F layer and reasons for convection in the Earth's core, Doklady Akad. Nauk SSSR, 149, 8-10, 1963.

De Groot, S. R. and P. Mazur, Non-Equibrium Thermodynamics, 510 pp., North-Holland Pub. Co., 1962.

Einstein, A., Eine neue Bestimmung der Molekul-dimensionen, Ann. Phys., 19, 289-306, 1906.

FEARN, D. R. and D. S. LOPER, Compositional convection and stratification of Earth's core, Nature, 289, 393-394, 1981. 
FrancK, S., Ascending droplets in the Earth's core, Phys. Earth Planet. Inter., 27, 249-254, 1982.

Gubbins, D., Energetics of the Earth's core, J. Geophys., 43, 453-464, 1977.

Hewitt, J. M., D. P. McKenzie, and N. O. Weiss, Dissipative heating in convective flows, J. Fluid Mech., 68, $721-738,1975$.

Ito, E., K. Morooka, T. Katsura, and O. Ujinke, Reaction between molten iron and silicate melts at high pressure and temperature, in Abstract of the Third SEDI Symposium, 21, 1992.

JacoBs, J. A., The Earth's Core, 253 pp., Academic Press, 1975.

KATO, T. and A. E. Ringwood, Melting relationships in the system Fe-FeO at high pressures: implications for the composition and Formation of the Earth's core, Phys. Chem. Minerals, 16, 524-538, 1989.

Kimura, R., Cell formation in the convective mixed layer, Fluid Dyn. Res., 3, 395-399, 1988.

Koyaguchi, T., M. A. Halloworth, H. E. Huppert, and R. S. J. Sparks, Sedimentation of particles from a convective fluid, Nature, 343, 447-450, 1990.

LOPER, D. S., The gravitationally powered dynamo, Geophys. J. R. Astron. Soc., 54, 389-404, 1978.

LOPER, D. S. and P. H. ROBERTS, Compositional convection and the gravitationally powered dynamo, in The Fluid Mechanics of Astrophysics and Geophysics (vol. 2 Stellar and planetary magnetism), edited by A. M. Soward, pp. 297-327, Gordon and Breach, 1983.

Metchnik, V. I., M. T. Gladwin, and F. D. StaCEy, Core convection as a power source for the geomagnetic dynamo-a thermodynamic argument, J. Geomag. Geoelectr., 26, 405-415, 1974.

Moffatt, H. K., Liquid metal MHD and the geodynamo, in Proceedings of the IUTAM Symposium on Liquid Metal Magnetohydrodynamics, 403 pp., Kluwer Academic, 1989.

Ohtani, E. and A. E. Ringwood, Composition of the core, I. Solubility of oxygen in molten iron at high temperatures, Earth Planet. Sci. Lett., 71, 85-93, 1984.

PoIRIER, J. P., Transport properties of liquid metals and viscosity of the Earth's core, Geophys. J., 92, 99-105, 1988.

UrakaWA, S., M. Kato, and M. KumazaWA, Experimental study on the phase relations in the system Fe-Ni-O-S to $15 \mathrm{GPa}$, in High-Pressure Research in Mineral Physics, edited by M. H. Manghnani and Y. Syono, pp. 95-111, A.G.U., 1987.

Verhoogen, J., Thermal regime of the Earth's core, Phys. Earth Planet. Inter., 7, 47-58, 1973. 